Pacific Journal of Mathematics

DETERMINATION OF THE INTERTWINING OPERATORS FOR
HOLOMORPHICALLY INDUCED REPRESENTATIONS OF

Brian bot, Thomas Jones Enright and Brad Shelton 


\title{
DETERMINATION OF THE INTERTWINING OPERATORS FOR HOLOMORPHICALLY INDUCED REPRESENTATIONS OF HERMITIAN SYMMETRIC PAIRS
}

\author{
Brian D. Boe, Thomas J. EnRIght AND Brad Shelton
}

Let $X$ and $Y$ be two holomorphically induced representations for an irreducible Hermitian symmetric pair $(G, K)$ with integral highest weights. Then $\operatorname{Hom}(X, Y)$ equals either $\mathbf{C}$ or zero. In this article we give formulas for $\operatorname{Hom}(X, Y)$ when the infinitesmial character of $X$ and $Y$ is integral and either regular or semi-regular. One formula is given in terms of the highest weight of $X$ and the other in terms of the highest weight of $Y$.

1. Introduction. Let $X$ and $Y$ be two holomorphically induced representations for an irreducible Hermitian symmetric pair $(G, K)$ with integral highest weights. Then $\operatorname{Hom}(X, Y)$ equals either $\mathbf{C}$ or zero ([2], [7]). In this article we give formulas for $\operatorname{Hom}(X, Y)$ when the infinitesimal character of $X$ and $Y$ is integral and either regular or semi-regular. One formula is given in terms of the highest weight of $X$ and the other in terms of the highest weight of $Y$. These formulas were established for the case of $\mathrm{SU}(p, q)$ by the first two authors [3].

This article complements the results of [7] which include formulas for the composition factors of the modules $X$ and $Y$ above when $G$ is of classical type. With this in mind we shall suppose the reader is familiar with the notation and results of [7], especially sections eight through thirteen of that article. Since [7] does not include formulas for the composition factors when $G$ is of exceptional type we will provide formulas in those cases. These formulas will be consistent with the formulas of [7] and will be derived with the help of [4].

Recently, $\mathrm{H}$. Jakobsen has given a formula for $\operatorname{Hom}(X, Y)$ when one of these modules is induces from a one dimensional module [9]. Our formula for $\operatorname{Hom}\left(N_{y}, N_{x}\right)$ below follows from Jakobsen's work in the cases where either $x$ is the identity element or $y$ is the element of maximal length in $\mathscr{W}^{\mathfrak{m}}$. Otherwise our results are disjoint.

For references to other work on the description of $\operatorname{Hom}(X, Y)$ the reader should consult the introductions and bibliographies in [2] and [7]. 
2. Statement of results. In order to state our results we recall some of the notation of [7]. Let $g$ (resp. $m$ ) be the complexified Lie algebra of $G$ (resp. $K$ ), and let $\mathfrak{p}$ be a maximal parabolic subalgebra of $g$ with Levi component $\mathfrak{m}$ and nilradical $\mathfrak{u}$. Let $\mathfrak{h}$ be a Cartan subalgebra of $\mathfrak{g}$ and $\mathfrak{m}$ and let $\mathfrak{b}$ be a Borel subalgebra of $\mathfrak{g}$ with $\mathfrak{h} \subseteq \mathfrak{b} \subseteq \mathfrak{p}$. The Weyl group of $\mathfrak{g}($ resp. $\mathfrak{m})$ is $\mathscr{W}\left(\right.$ resp. $\left.\mathscr{W}_{\mathrm{m}}\right)$ and we have $\mathscr{W}=\mathscr{W}_{\mathrm{m}} \mathscr{W}^{\mathfrak{m}}$ where $\mathscr{W}^{\mathrm{m}}$ is the set of minimal length left coset representations. The projection of $\mathscr{W}$ onto $\mathscr{W}^{\mathrm{m}}$ given by the decomposition $\mathscr{W}=\mathscr{W}_{\mathrm{m}} \mathscr{W}^{\mathrm{m}}$ is denoted by $\omega \mapsto \bar{\omega}$. Let $\Delta, \Delta(\mathfrak{m})$ and $\Delta(\mathfrak{u})$ be the sets of $\mathfrak{h}$-roots of $\mathfrak{g}, \mathfrak{m}$ and $\mathfrak{u}$ respectively. Put $2 \rho=\Sigma_{\alpha \in \Delta^{+}} \alpha$.

For $\lambda \in \mathfrak{h}^{*}$ we denote by $M(\lambda)$ the $\mathrm{g}$-Verma module of highest weight $\lambda-\rho$. Then $L(\lambda)$ and $N(\lambda)$ denote the simple quotient and maximal m-locally finite quotient of $M(\lambda)$ respectively (cf. [7]). For $x \in \mathscr{W}^{\mathfrak{m}}$ we denote by $N_{x}$ and $L_{x}$ the modules $N(x \rho)$ and $L(x \rho)$. We adopt the convention that if all roots are the same length, they are called short.

Definition 2.1. Set $\mathscr{M}=\left\{(\gamma, \nu) \mid \gamma, \nu \in \Delta^{+}\right.$and either $\langle\gamma, \nu\rangle \neq 0$ or both $\gamma$ and $\nu$ are long roots $\}$. Let $\mathscr{S}\left(\Delta^{+}\right)$denote the collection of all subsets $\Omega$ of $\Delta^{+}$which satisfy the following conditions:

(a) If $\gamma, \nu$ are in $\Omega, \gamma \neq \nu$, then $(\gamma, \nu) \notin \mathscr{M}$.

(b) If $\gamma$ is in $\Omega$ and $\xi$ is in $\Delta^{+}$with $\gamma \neq \xi,(\gamma, \xi) \in \mathscr{M}$ and $\xi \leq \gamma$ then there is a $\zeta$ in $\Omega$ with $\zeta \neq \gamma,(\zeta, \xi) \in \mathscr{M}$ and $\zeta \leq \gamma$.

We note that $\mathscr{S}\left(\Delta^{+}\right)$is defined for any positive system of any root system. When there is no chance of confusion we will denote this set simply as $\mathscr{S}$.

Fix $x$ in $\mathscr{W}^{\mathrm{m}}$. Then $\mathscr{S}_{x}$ will denote the set of all $\Omega$ in $\mathscr{S}$ which satisfy $x \Omega \subseteq \Delta(\mathfrak{u}) \cup-\Delta(\mathfrak{u})$. By $\mathscr{E}_{x}$ we will denote the collection of $\Omega$ in $\mathscr{S}_{x}$ which satisfy the additional condition:

(c) If $\gamma$ is in $\Omega$ then there is a $\zeta$ in $\Omega$ with $\gamma \leq \zeta$ and $x \zeta \in \Delta(\mathfrak{u})$.

For $\Omega \in \mathscr{S}_{x}$, put $\Omega^{+}=\{\gamma \in \Omega \mid x \gamma \in \Delta(\mathfrak{u})\}$ and $\Omega^{-}=\{\gamma \in \Omega \mid x \gamma \in$ $-\Delta(\mathfrak{u})\}$. We say that $\Omega$ is $x$-positive (resp. $x$-negative) if $\Omega=\Omega^{+}$(resp. $\Omega=\Omega^{-}$). Note that the $x$-positive elements of $\mathscr{S}_{x}$ are automatically in $\mathscr{E}_{x}$. Let $r_{\Omega}=\prod_{\gamma \in \Omega^{+}} s_{\gamma}$ and $t_{\Omega}=\prod_{\gamma \in \Omega^{-}} s_{\gamma}$.

We can now state our main result.

Proposition 2.2. Let $x$ and $y$ be in $\mathscr{W}^{m}$. Then:

(a)

$$
\operatorname{Hom}\left(N_{y}, N_{x}\right)= \begin{cases}\mathbf{C} & \text { if } y=\overline{x r_{\Omega}} \text { for some } x \text {-positive } \Omega \text { in } \mathscr{S}_{x}, \\ 0 & \text { otherwise. }\end{cases}
$$


(b)

$$
\operatorname{Hom}\left(N_{y}, N_{x}\right)= \begin{cases}\mathbf{C} & \text { if } x=\overline{y t_{\Omega}} \text { for some } y \text {-negative } \Omega \text { in } \mathscr{S}_{y}, \\ 0 & \text { otherwise. }\end{cases}
$$

We will prove only part (a) of (2.2). The proof of (b) is exactly the same and we omit the details.

In order for the reader to better relate (2.2) to the theory of composition factors we recall the following proposition from [7].

Proposition 2.3. Assume that $\mathrm{g}$ is of classical type and let $x$ and $y$ be in $\mathscr{W}^{\mathrm{m}}$. Then

(a) The mappings $\Omega \mapsto \Omega^{+}$and $\Omega \mapsto \overline{x r_{\Omega}}$ are injective when restricted to $\mathscr{E}_{x}$

(b) The simple module $L_{y}$ is a composition factor of $N_{x}$ if and only if $y=\overline{x r_{\Omega}}$ for some $\Omega$ in $\mathscr{E}_{x}$. Moreover, the composition factors of $N_{x}$ occur with multiplicity one.

We will verify in section four that (2.3) also holds when $g$ is of exceptional type (cf. (4.3)).

3. The classical cases. Assume that $g$ is of classical type. Let notation be as in [7], sections eight through thirteen. There are five classical cases to consider which we denote by HS. $i, 1 \leq i \leq 5$ [7, Table 8.1]. These correspond respectively to the cases: $\mathrm{SU}(p, q), \mathrm{SO}(2 n-1,2), \mathrm{Sp}(2 n, \mathbf{R})$, $\mathrm{SO}(2 n-2,2)$ and $\mathrm{SO}^{*}(2 n)$. In the cases of HS. 2 and HS. 4 the result can be obtained directly from $[6, \S 7]$ and $[6, \S 9]$ respectively. If $(\mathfrak{g}, \mathfrak{p})$ is of type HS.1, HS.3 or HS.5 with constant $p$ equal to one $[7,(8.1)]$ then the result follows from $[6, \S 6]$. So we may assume that $(\mathfrak{g}, \mathfrak{p})$ is of type HS.1, HS. 3 or HS.5 with constant $p>1$. We proceed by induction on the constant $p$. If $\alpha$ is a simple root we put $\mathscr{W}_{\alpha}=\left\{x \in \mathscr{W}^{\mathfrak{m}} \mid x \alpha \in-\Delta(\mathfrak{u})\right\}$. Let $\omega_{\alpha}$ be the fundamental weight corresponding to $\alpha$. For $x$ or $x s_{\alpha}$ in $\mathscr{W}_{\alpha}$, write $N_{x}^{\alpha}$ and $L_{x}^{\alpha}$ for $N\left(x\left(\rho-\omega_{\alpha}\right)\right)$ and $L\left(x\left(\rho-\omega_{\alpha}\right)\right)$ respectively.

LEMMA 3.1. Let $\alpha$ be a simple root and let $x, y \in \mathscr{W}_{\alpha}$. Then:

(a) $\operatorname{Hom}\left(N_{y}^{\alpha}, N_{x}^{\alpha}\right) \cong \mathbf{C}$ or zero depending as $y=\overline{x r_{\Omega}}$ for some $x$-positive set $\Omega$ in $\mathscr{S}_{x, \alpha}$ or not.

(b) $\operatorname{Hom}\left(N_{y}^{\alpha}, N_{x}^{\alpha}\right) \cong \mathbf{C}$ or zero depending as $x=\overline{y t_{\Omega}}$ for some $y$-negative set $\Omega$ in $\mathscr{S}_{y, \alpha}$ or not. 
Proof. Suppose that $\alpha$ is a short root and let $\Lambda_{\alpha}: \mathcal{O}^{\prime} \rightarrow \mathcal{O}_{\alpha}$ be the equivalence of categories guaranteed by [7, (10.1) and (11.2)]. The inductive hypotheses assure that (2.2) holds in the category $\mathcal{O}^{\prime}$. Thus (3.1) follows from the formulas of [7, (10.5) and (11.7)].

If $\beta$ is the long simple root of HS.3 then let $\alpha$ be the adjacent short simple root. (3.1) holds for $\alpha$; and so, by the formulas of [7, (12.15), (12.16) and (12.17)] and the equivalence of categories in [7, (12.14)], (3.1) must also hold for $\beta$. This completes (3.1).

In [2], an algorithm was given for computing $\operatorname{Hom}\left(N_{y}, N_{x}\right)$ inductively, using [7, Proposition 11.2]. This algorithm is the main ingredient in the remaining part of our proof. For $x$ and $y$ in $\mathscr{W}^{\mathfrak{m}}$ set $d(y, x)=$ $\operatorname{dim}\left(\operatorname{Hom}\left(N_{y}, N_{x}\right)\right)$. We know from [2] or [7] that $d(y, x) \leq 1$. If $x$ has maximal length in $\mathscr{W}^{\mathrm{m}}$ then $N_{x}=L_{x}$, there are no nonempty $x$-positive sets in $\mathscr{S}_{x}$ and $d(y, x) \neq 0$ if and only if $x=y$. Thus we may assume that $x$ does not have maximal length.

Fix a nonempty $x$-positive set $\Omega$ in $\mathscr{E}_{x}$ and set $y=\overline{x r_{\Omega}}$. By [8, proof of (4.2)], if $\Omega$ is a set of simple roots then the standard map from $N_{y}$ to $N_{x}$ is nonzero and $d(y, x)=1$. Thus we assume that $\Omega$ has some non-simple roots.

Choose $\gamma$ to be any root in $\Omega$ that is minimal with respect to $\leq$ among the non-simple roots of $\Omega$. We claim that there is short simple root $\beta$ with

$$
\left\langle\beta, \gamma^{\vee}\right\rangle=1 .
$$

If $\gamma$ is of the form $e_{l}-e_{j}$ then set $\beta=e_{l}-e_{i+1}$. If $\gamma=e_{i}+e_{j}$ with $i \leq j<n$ then set $\beta=e_{j}-e_{j+1}$. If $\gamma=e_{i}+e_{n}, 1 \leq i<n-1$, then set $\beta=e_{t}-e_{t+1}$. Finally, suppose that $\gamma=e_{n-1}+e_{n}$. Since $\gamma$ is not simple, $(\mathfrak{g}, \mathfrak{p})$ must be of type HS.3. Set $\lambda=2 e_{n}$. Then $(\lambda, \gamma) \in \mathscr{M}$ and $\lambda \leq \gamma$. Thus, by (2.1), there is an $\alpha \in \Omega$ with $\alpha \neq \gamma$ and $\alpha \leq \gamma$. This forces $\alpha$ to be $e_{n-1}-e_{n}$. This contradicts the assumption that $\Omega$ is in $\mathscr{E}_{x}$ since $x \alpha$ and $x \gamma$ cannot both be in $\Delta(\mathfrak{u}) \cup-\Delta(\mathfrak{u})$. Thus the final case does not occur. This proves the claim.

Let $\beta$ be as in (3.2). Then $\beta \leq \gamma$; and so by (2.1), there is an $\alpha$ in $\Omega$ with $\alpha \leq \gamma, \alpha \neq \gamma$ and $(\alpha, \beta) \in \mathscr{M}$. Minimality of $\gamma$ forces $\alpha$ to be simple. Thus, since $\beta$ is a short root, $\alpha$ and $\beta$ are adjacent simple roots.

Lemma 3.3. Let $\Omega, \alpha, \beta$ and $\gamma$ be as above. Then $\alpha$ and $\gamma$ are the only roots in $\Omega$ not orthogonal to $\beta$.

Proof. Since $x \alpha \in \Delta(\mathfrak{u})$ and $\left\langle x \alpha^{\vee}, x \beta\right\rangle=-1, x \beta \notin \Delta(\mathfrak{u})$. Similarly, since $x \gamma \in \Delta(\mathfrak{u})$ and $\left\langle x \gamma^{\vee}, x \beta\right\rangle=1, x \beta \notin-\Delta(\mathfrak{u})$. Thus $x \beta \in \Delta(\mathfrak{m})$. 
Let $\mu$ be the unique simple root in $\Delta(\mathfrak{u})$. Let $\zeta$ be any third root in $\Omega$. Then, $x \zeta \in \Delta(\mathfrak{u})$. However, $x s_{\alpha} s_{\zeta} \beta=x \beta+x \alpha-\left\langle\beta, \zeta^{\vee}\right\rangle x \zeta$ cannot have $\mu$-coefficient larger than 1 ; and so, $\left\langle\beta, \zeta^{\vee}\right\rangle \geq 0$. Similarly, $x s_{\gamma} s_{\zeta} \beta=x \beta$ $-x \gamma-\left\langle\beta, \zeta^{\vee}\right\rangle x \zeta$ cannot have $\mu$-coefficient less than -1 ; and so, $\left\langle\beta, \zeta^{\vee}\right\rangle \leq 0$. Thus $\langle\beta, \zeta\rangle=0$. This proves the lemma.

From the lemma we see that $y \beta=\overline{x r_{\Omega}} \beta=m x s_{\alpha} s_{\gamma} \beta=m\left(x s_{\alpha} \beta-x \gamma\right)$ where $m \in \mathscr{W}_{\mathrm{m}}$. Also, $x s_{\alpha} \beta=x(\alpha+\beta)$. Since $x \alpha, x \gamma \in \Delta(\mathfrak{u})$, we have exactly two possibilities,

$$
x s_{\alpha} \beta \in \Delta(\mathfrak{m}) \text { and } y \beta \in-\Delta(\mathfrak{H})
$$

or

$$
x s_{\alpha} \beta \in \Delta(\mathfrak{u}) \text { and } y \beta \in \Delta(\mathfrak{m}) .
$$

In each of these cases, by [2, (2.3)], $d\left(y, x s_{\alpha}\right)=0$. The short exact sequence

$$
0 \rightarrow N_{x} \rightarrow \phi_{\alpha} N_{x}^{\alpha} \rightarrow N_{x s_{\alpha}} \rightarrow 0
$$

gives rise to the long exact sequence

$$
0 \rightarrow \operatorname{Hom}\left(N_{y}, N_{x}\right) \rightarrow \operatorname{Hom}\left(N_{y}, \phi_{\alpha} N_{x}^{\alpha}\right) \rightarrow \operatorname{Hom}\left(N_{y}, N_{x s_{\alpha}}\right) \rightarrow \cdots .
$$

We have seen that the last term here is zero. Combining this with (3.1) and the adjoint property of $\phi_{\alpha}$ and $\psi_{\alpha}$ we see $d(y, x)=\operatorname{dim}\left(\operatorname{Hom}\left(\psi_{\alpha} N_{y}, N_{x}^{\alpha}\right)\right)$ $=\operatorname{dim}\left(\operatorname{Hom}\left(N_{y}^{\alpha}, N_{x}^{\alpha}\right)\right)=1$.

Conversely, suppose now that $d(y, x)=1$. Then by (2.3) there is an $\Omega$ in $\mathscr{E}_{x}$ with $y=\overline{x r_{\Omega}}$. We must show that $\Omega$ is $x$-positive. Let $\alpha$ be a simple root in $\Omega$. Then $y \alpha \in-\Delta(\mathfrak{u})$, so there is a surjection $\phi_{\alpha} N_{y}^{\alpha} \rightarrow N_{y}$ $\rightarrow 0$. This gives an injection $0 \rightarrow \operatorname{Hom}\left(N_{y}, N_{x}\right) \rightarrow \operatorname{Hom}\left(N_{y}^{\alpha}, N_{x}^{\alpha}\right)$. Thus by (3.1), $\Omega \backslash\{\alpha\}$ is an $x$-positive set in $\mathscr{S}_{x, \alpha}$. It remains only to show: $\alpha \in \Omega^{+}$. Suppose $\alpha \in \Omega^{-}$, i.e. $x \alpha \in-\Delta(\mathfrak{u})$. If $\mu$ is any other simple root in $\Omega$ then, as above, $\Omega \backslash\{\mu\}$ is $x$-positive and thus $x \alpha \in \Delta(\mathfrak{u})$. This contradiction assures that $\alpha$ is the unique simple root in $\Omega$. Set $z=x s_{\alpha}$. Then $\Omega$ is a $z$-positive element of $\mathscr{E}_{z}$ and $y=\overline{z \prod_{\gamma \in \Omega} s_{\gamma}}$. But then, by the preceding paragraph, $d(y, z)=1$ and $d\left(y, z s_{\alpha}\right)=0$. However, $d\left(y, z s_{\alpha}\right)$ $=d(y, x)=1$. This contradiction shows that $\alpha$ must lie in $\Omega^{+}$and completes the proof of (2.2) in the classical cases.

4. The exceptional cases. Here we verify (2.2) when $g$ is of exceptional type $\mathrm{E}_{6}$ or $\mathrm{E}_{7}$. To make sense of (2.2) in the exceptional cases we must first prove a generalization of (2.3) for these cases.

For $x, y \in \mathscr{W}^{\mathfrak{m}}$ write $\left(N_{x}, L_{y}\right)$ for the multiplicity of $L_{y}$ as a composition factor of $N_{x}$. We write $F_{x}$ for the set of all $y \in \mathscr{W}^{\mathfrak{m}}$ with $\left(N_{y}, L_{x}\right)=1$. The labeled posets $\mathscr{W}^{\mathrm{m}}$ for the two exceptional cases are given in Figures 4.1 and 4.2. These are consistent with the notation of [4] 

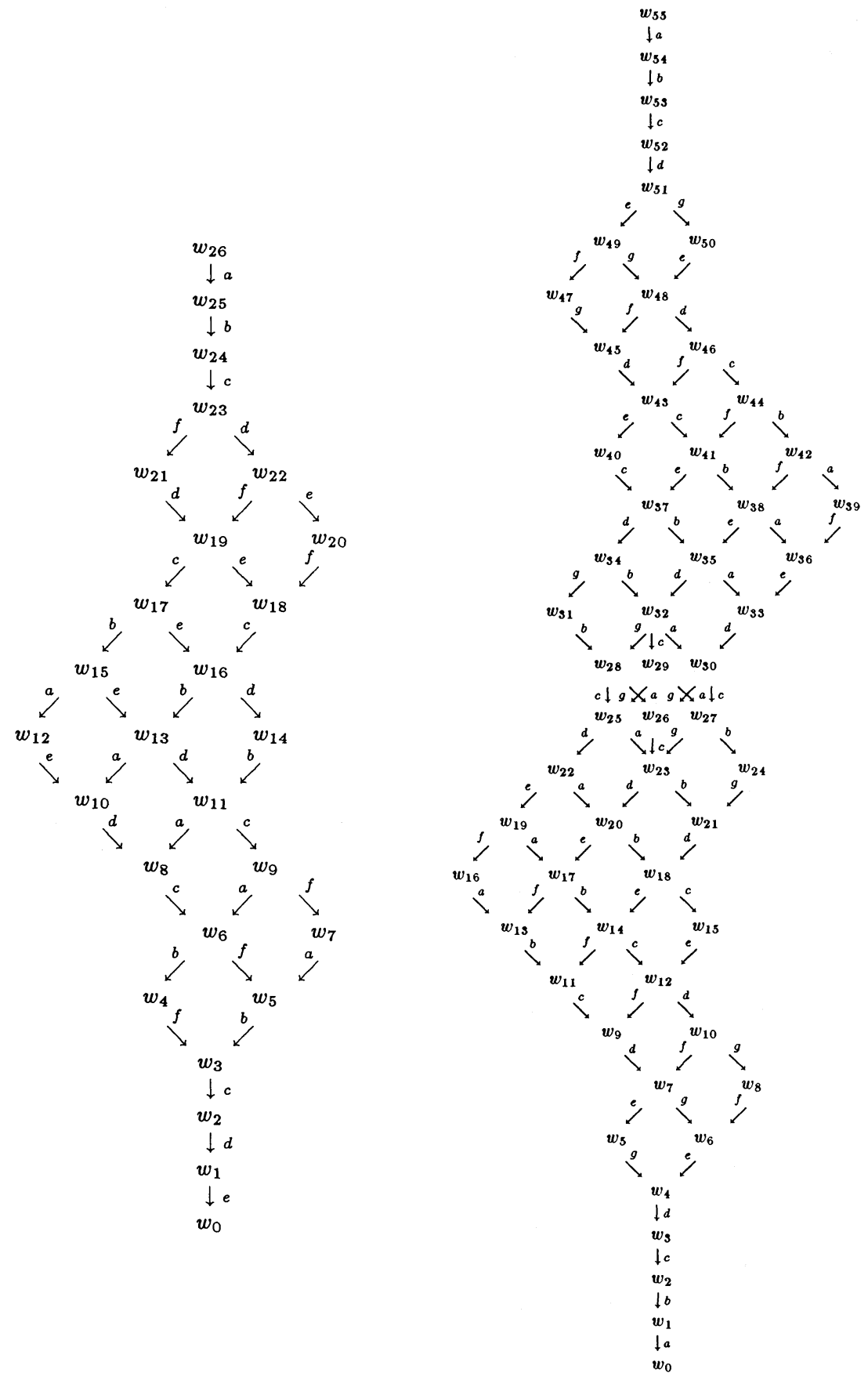

FIGURE 4.1. $\mathscr{W}^{\mathrm{m}}$ for $\mathrm{E}_{6}$

FIGURE 4.2. $\mathscr{W}^{\mathrm{m}}$ for $\mathrm{E}_{7}$ 
and the labelings of the posets are consistent with the following labelings of the Dynkin diagrams:

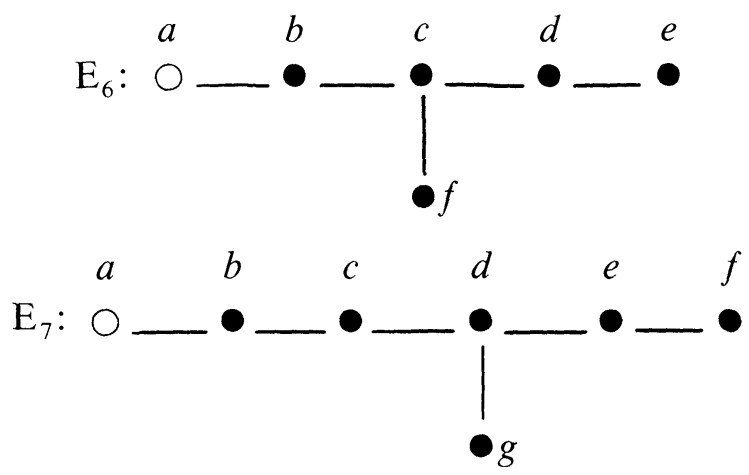

In the labeled posets, the notation $w_{l} \stackrel{\alpha}{\rightarrow} w_{J}$ means that $\alpha$ is a simple root, $w_{i}=w_{l} s_{\alpha}$ and $w_{j} \in \mathscr{W}_{\alpha}$. We will use the Bourbaki convention for denoting roots. Simple roots will be denoted by the letter which labels them in the Dynkin diagram (4.1) or (4.2). In $\mathrm{E}_{6}$,

$$
\left(\begin{array}{lllll}
r_{a} & r_{b} & r_{c} & r_{d} & r_{e} \\
& & r_{f} &
\end{array}\right)=r_{a} a+r_{b} b+r_{c} c+r_{d} d+r_{e} e+r_{f} f .
$$

In $\mathrm{E}_{7}$,

$$
\left(\begin{array}{llllll}
r_{a} & r_{b} & r_{c} & r_{d} & r_{e} & r_{f} \\
& & & r_{g} & &
\end{array}\right)=r_{a} a+r_{b} b+r_{c} c+r_{d} d+r_{e} e+r_{f} f+r_{g} g .
$$

Recall from [7, (8.3)] the definition of the orthogonal sets of noncompact positive roots $\Sigma_{x}$ and $\Sigma_{x}^{+}$for each $x \in \mathscr{W}^{\mathrm{m}}$. For any orthogonal set $\Omega$ contained in $\Delta(\mathfrak{u})$ set $s_{\Omega}=\prod_{\gamma \in \Omega} s_{\gamma}$. Recall that if $\alpha$ is any simple root and $x \in \mathscr{W}_{\alpha}$ then $\Sigma_{x, \alpha}=\Sigma_{x} \backslash\{-x \boldsymbol{\alpha}\}$ and $\Sigma_{x, \alpha}^{+}=\Sigma_{x s_{\alpha}}^{+} \backslash\{-x \alpha\}$. Recall also the definition of the sets $\mathscr{S}_{x, \alpha}$ from $[7,(9.4)]$.

Proposition 4.3. Let $(\mathfrak{g}, \mathfrak{p})$ be of exceptional type $\mathrm{E}_{6}$ or $\mathrm{E}_{7}$ and let $x$ and $y$ be in $\mathscr{W}^{\mathrm{m}}$. Then

(a) $-x^{-1} \Sigma_{x}$ is in $\mathscr{S}_{x}$.

(b) $\left(N_{v}, L_{x}\right)=1$ if and only if $y=\overline{s_{\Omega} x}$ for some $\Omega \subseteq \Sigma_{x}$.

(c) $\left(N_{v}, L_{x}\right)=1$ if and only if $x=\overline{x r_{\Omega}}$ for some $\Omega \in \mathscr{E}_{y}$.

(d) ([1]) The composition factors of $N_{x}$ occur with multiplicity one.

(e) $x^{-1} \Sigma_{x}^{+}$is in $\mathscr{S}_{x}$.

Proof. We begin by proving parts (a) and (b). Choose $x \in \mathscr{W}_{f}$ (cf. (4.1) and (4.2)). In Tables 4.1 and 4.2 we have listed $-x^{-1} \Sigma_{x}$ and all those $N_{y}$ in which $L_{x}$ appears as a composition factor, i.e. $F_{x}$. The computation of $-x^{-1} \Sigma_{x}$ has been done directly and the composition factors are taken from [4]. We claim that for each $x \in \mathscr{W}_{f},-x^{-1} \Sigma_{x}$ is in $\mathscr{S}_{x}$. It suffices only 
TABLE 4.1

$\mathrm{E}_{6}$ data for $w_{J} \in \mathscr{W}_{f}$. The sets $-w_{j}^{-1} \Sigma_{w_{j}}$ and $F_{j}=\left\{i \mid\left(N_{w_{i}}, L_{w_{j}}\right)=1\right\}$.

\begin{tabular}{|c|c|c|}
\hline$j$ & $-w_{j}^{-1} \Sigma_{w,}$ & $F_{j}$ \\
\hline 21 & $f$ & 23,21 \\
19 & $f, d$ & $23,22,21,19$ \\
18 & $f, e$ & \\
$22,20,19,18$ \\
7 & $f,\left(\begin{array}{rrrr}0 & 1 & 2 & 0 \\
& 1 & \end{array}\right)$ & $20,18,9,7$ \\
5 & $f, a$ & $9,7,6,5$ \\
3 & $f, b$ & $6,5,4,3$ \\
\hline
\end{tabular}

\section{TABLE 4.2}

Case $\mathrm{E}_{7}$ data for $w_{J} \in \mathscr{W}_{f}$. The sets $-w_{j}^{-1} \Sigma_{w_{J}}$ and $F_{J}=\left\{i \mid\left(N_{w_{t}}, L_{w_{J}}\right)=1\right\}$.

\begin{tabular}{|c|c|c|c|c|}
\hline$j$ & & $-w_{j}^{-1} \Sigma_{w}$ & & $F_{j}$ \\
\hline 47 & & $f$ & & 49,47 \\
\hline 45 & & $f, g$ & & $49,48,47,45$ \\
\hline 43 & & $f, d$ & & $48,46,45,43$ \\
\hline 41 & & $f, c$ & & $46,44,43,41$ \\
\hline 38 & & $f, b$ & & $44,42,41,38$ \\
\hline 36 & & $f, a$ & & $42,39,38,36$ \\
\hline 16 & $f,($ & 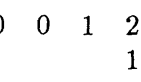 & $\left.\begin{array}{ll}2 & 1 \\
& \end{array}\right)$ & $42,38,19,16$ \\
\hline 13 & $f, a$ & $\begin{array}{lll}0 & 0 & 1\end{array}$ & $\left.\begin{array}{lll}2 & 2 & 1 \\
1 & & \end{array}\right)$ & $42,39,38,36,19,17,16,13$ \\
\hline 11 & $f, b$, & $\begin{array}{lll}1 & 1 & 1\end{array}$ & $\left.\begin{array}{lll}2 & 2 & 1 \\
1 & & \end{array}\right)$ & $44,42,41,38,17,14,13,11$ \\
\hline 9 & $f, c$, & $\begin{array}{lll}1 & 2 & 2\end{array}$ & $\left.\begin{array}{lll}2 & 2 & 1 \\
1 & & \end{array}\right)$ & $46,44,43,41,14,12,11,9$ \\
\hline 7 & $f, d$, & $\begin{array}{lll}1 & 2 & 3\end{array}$ & $\left.\begin{array}{lll}3 & 2 & 1 \\
1 & & \end{array}\right)$ & $48,46,45,43,12,10,9,7$ \\
\hline 6 & $f, g$ & $\begin{array}{lll}1 & 2 & 3\end{array}$ & $\left.\begin{array}{lll}4 & 2 & 1 \\
2 & & \end{array}\right)$ & $49,48,47,45,10,8,7,6$ \\
\hline
\end{tabular}

to show that $-x^{-1} \Sigma_{x}$ satisfies (2.1b). Choose $\xi \in \Delta^{+}$and $\gamma \in-x^{-1} \Sigma_{x}$ with $\xi \leq \gamma, \xi \neq \gamma$ and $\langle\xi, \gamma\rangle \neq 0$. Then $\gamma$ cannot be simple; and so, by Tables 4.1 and 4.2, either $\mathrm{g}$ is $\mathrm{E}_{6}$ and $x=w_{7}$ or $\mathrm{g}$ is $\mathrm{E}_{7}$ and $x=w_{j}$ with $j \in\{6,7,9,11,13,16\}$. A case by case check in these seven cases shows that (2.1b) holds. For each $x \in \mathscr{W}_{f}$ we see from Tables 4.1 and 4.2 that $\operatorname{card}\left(F_{x}\right)=2^{\operatorname{card}\left(\Sigma_{1}\right)}$. Thus, to prove $(4.3 \mathrm{~b})$ for $x \in \mathscr{W}_{f}$ it suffices to observe that for each $\Omega \subseteq \Sigma_{x}, \overline{s_{\Omega} x} \in F_{x}$. This computation can be verified from the tables.

Now fix any $x \in \mathscr{W}^{\mathrm{m}}$. If $x=e$ then $\Sigma_{x}=\varnothing$ and $F_{x}=\{x\}$. So assume that $x \neq e$. Then there is a simple root $\alpha$ with $x \in \mathscr{W}_{\alpha}$. If $\alpha=f$ 
then we have already seen that (4.3a) and (4.3b) hold for $x$. So assume that $\alpha \neq f$. By a theorem of Vogan, [10], or more specifically [7, (10.1)], there is an equivalence of categories $\pi: \mathcal{O}_{\alpha} \rightarrow \mathcal{O}_{f}$. Define $w \in \mathscr{W}_{f}$ by $\pi\left(L_{x}\right) \cong L_{w}$. Then by (4.3a) and (4.3b) for $w,-w^{-1} \Sigma_{w, f} \in \mathscr{S}_{w, f}$ and $F_{w} \cap \mathscr{W}_{f}=\left\{\overline{s_{\Omega} w} \mid \Omega \subseteq \Sigma_{w, f}\right\}$. It follows from [7, (10.4) and (10.5)] that $-x^{-1} \Sigma_{x, \alpha} \in \mathscr{S}_{x, \alpha}$ and $F_{x} \cap \mathscr{W}_{\alpha}=\left\{\overline{s_{\Omega} x} \mid \Omega \subseteq \Sigma_{x, \alpha}\right\}$. Thus, by [7, (9.5)], $-x^{-1} \Sigma_{x} \in \mathscr{S}_{x}$. We also see, exactly as in [7, (12.4)], that $F_{x}=\left\{\overline{s_{\Omega} x} \mid \Omega \subseteq\right.$ $\left.\Sigma_{x}\right\}$. This completes the proof of (4.3a) and (4.3b).

Part (c) follows from parts (a) and (b) and [7, (9.6)]. Part (d) comes from [1]. Finally, (e) follows from (a) and [5, (5.1)]. This proves (4.3).

The proof of (2.2) in the exceptional cases proceeds as in the classical cases. We need only establish (3.1) and (3.2) for the exceptional cases. These will be (4.5) and (4.6) (respectively) below. For any $x \in \mathscr{W}^{\mathfrak{m}}$, put $H_{x}=\left\{y \in \mathscr{W}^{\mathfrak{m}} \mid \operatorname{Hom}\left(N_{y}, N_{x}\right) \cong \mathbf{C}\right\}$. Recall from [1] that we know $d(y, x) \leq 1$ for all $x, y \in \mathscr{W}^{\mathrm{m}}$. Moreover, from [2], the sets $H_{x}$ are known explicitly. We wish only to show:

$$
H_{x}=\left\{\overline{x r_{\Omega}} \mid \Omega \text { an } x \text {-positive set in } \mathscr{S}_{x}\right\} \text {. }
$$

LEMMA 4.5. Let $\alpha$ be a simple root and let $x, y \in \mathscr{W}_{\alpha}$. Then $\operatorname{Hom}\left(N_{y}^{\alpha}, N_{x}^{\alpha}\right) \cong \mathbf{C}$ or zero depending as $y=\overline{x r_{\Omega}}$ for some $x$-positive set $\Omega$ in $\mathscr{S}_{x, \alpha}$ or not.

Proof. In Tables 4.3 and 4.4 we have listed the $x$-positive sets in $\mathscr{S}_{x, f}$ and the sets $H_{x, f}=\left\{y \in \mathscr{W}_{f} \mid \operatorname{Hom}\left(N_{y}^{f}, N_{x}^{f}\right) \neq 0\right\}$. The $x$-positive sets are found by direct computation while $H_{x, f}$ is gleaned from [2]. From the tables it follows by direct computation that (4.5) holds for $\alpha=f$. By [7, (10.1) and (10.5)], the lemma holds for all simple $\alpha$.

\section{TABLE 4.3}

$\mathrm{E}_{6}$ data for $w_{j} \in \mathscr{W}_{f}$. The $w_{j}$-positive sets in $\mathscr{S}_{w_{j}, f}$ and $H_{w_{j}, f}=\left\{i \mid w_{i} \in \mathscr{W}_{f}\right.$, $\left.\operatorname{Hom}\left(N_{w_{i}}^{f}, N_{w_{j}}^{f}\right) \neq 0\right\}$.

\begin{tabular}{|c|c|c|}
\hline$j$ & $w_{j}$-positive sets in $\mathcal{S}_{w_{j}}$ & $H_{w_{j}, f}$ \\
\hline 21 & $\emptyset,\{d\}$ & 21,19 \\
\hline 19 & $\emptyset,\{e\}$ & 19,18 \\
\hline 18 & $\emptyset,\left\{\left(\begin{array}{lllll}0 & 1 & 2 & 1 & 0 \\
& & 1 & & \end{array}\right)\right\}$ & 18,7 \\
\hline 7 & $\emptyset,\{a\}$ & 7,5 \\
\hline 5 & $\emptyset,\{b\}$ & 5,3 \\
\hline 3 & 0 & 3 \\
\hline
\end{tabular}




\section{TABLE 4.4}

$\mathrm{E}_{7}$ data for $w_{j} \in \mathscr{W}_{f}$. The $w_{j}$-positive sets in $\mathscr{S}_{w_{j}, f}$ and $H_{w_{j}, f}=\left\{i \mid w_{l} \in \mathscr{W}_{f}\right.$, $\left.\operatorname{Hom}\left(N_{w_{i}}^{f}, N_{w_{j}}^{f}\right) \neq 0\right\}$.

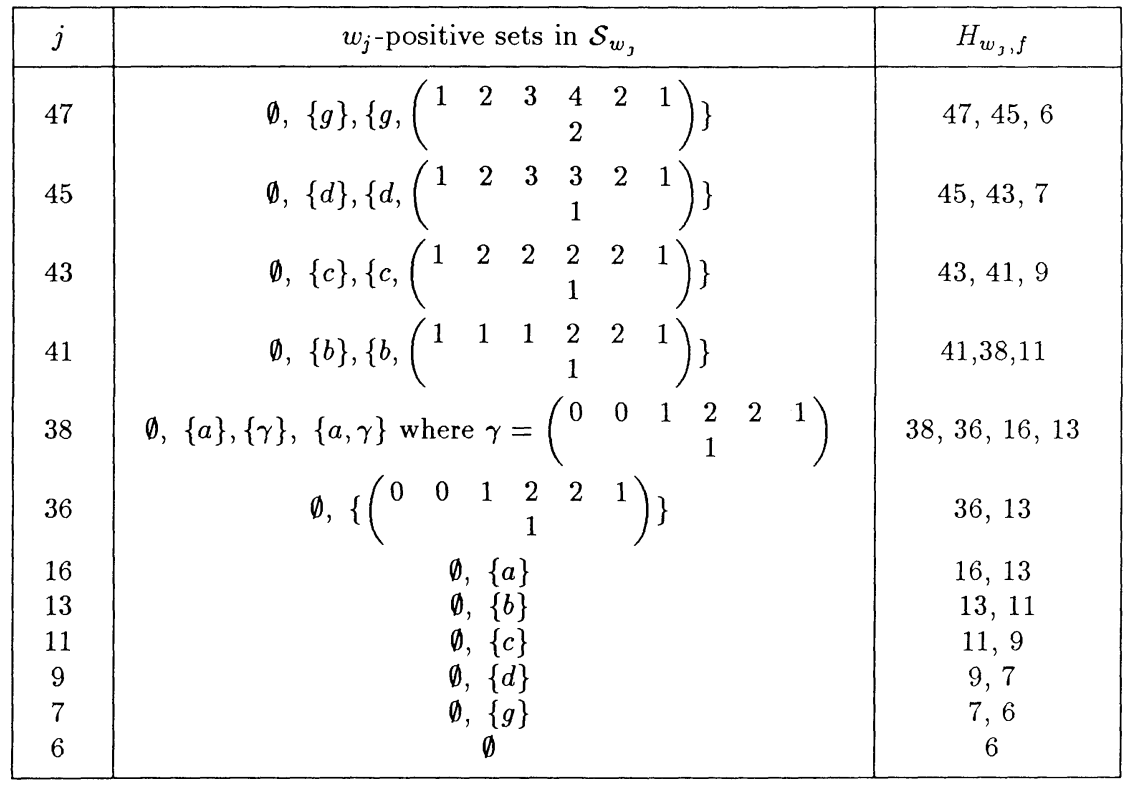

Let $\gamma$ be any non-simple positive root. We now claim:

There is a simple root $\beta$ with $\left\langle\beta, \gamma^{\vee}\right\rangle=1$.

Let $S$ be the set of simple roots and identify $S$ with the Dynkin diagram of g. Write $\gamma=\Sigma_{\mu \in S} r_{\mu} \mu$. Let $S_{\gamma}=\left\{\mu \in S \mid r_{\mu} \neq 0\right\}$. Then $S_{\gamma}$ forms a connected Dynkin subdiagram of $S$. Suppose that $\gamma$ satisfies the following condition.

There exist two adjacent simple roots $\beta$ and $\zeta$ with $r_{\beta}=r_{\zeta}=1$ and $\beta$ an extreme root of the diagram $S_{\gamma}$.

Then $\left\langle\beta, \gamma^{\vee}\right\rangle=1$ and $\beta \leq \gamma$, as required. Thus we are reduced to considering only those roots $\gamma$ which do not satisfy (4.7). These roots, together with appropriate $\beta$, are listed in Tables 4.5 and 4.6. In Table 4.6, which gives the necessary roots for $\mathrm{E}_{7}$, we have only included those roots for which $r_{a} \neq 0$. The $\mathrm{E}_{7}$ roots for which $r_{a}=0$ can be thought of as roots for $\mathrm{E}_{6}$ and are then included in Table 4.5. The tables complete the proof of (4.6). 
TABLE 4.5

$\mathrm{E}_{6}$ roots $\gamma$ which do not satisfy (4.7) and corresponding $\beta$ with $\left\langle\beta, \gamma^{\vee}\right\rangle$ $=1$.

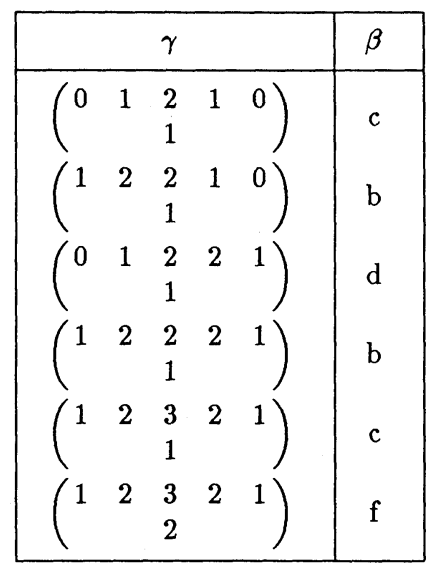

TABLE 4.6

$\mathrm{E}_{7}$ roots $\gamma$ with $r_{\alpha} \geq 1$ and which do not satisfy (4.7) and corresponding $\beta$ with $\left\langle\beta, \gamma^{\vee}\right\rangle=1$.

\begin{tabular}{|lllll|l|}
\hline \multicolumn{5}{|c|}{$\gamma$} & $\beta$ \\
\hline$\left(\begin{array}{llllll}1 & 2 & 2 & 2 & 1 & 0\end{array}\right)$ & $\mathrm{b}$ \\
$\left(\begin{array}{llllll}1 & 2 & 2 & 2 & 2 & 1\end{array}\right)$ & $\mathrm{b}$ \\
$\left(\begin{array}{llllll}1 & 2 & 2 & 3 & 2 & 1\end{array}\right)$ & $\mathrm{b}$ \\
$\left(\begin{array}{llllll}1 & 2 & 3 & 3 & 2 & 1\end{array}\right)$ & $\mathrm{c}$ \\
$\left(\begin{array}{llllll}1 & 2 & 2 & 3 & 2 & 1\end{array}\right)$ & $\mathrm{b}$ \\
$\left(\begin{array}{llllll}1 & 2 & 3 & 3 & 2 & 1\end{array}\right)$ & $\mathrm{c}$ \\
$\left(\begin{array}{llllll}1 & 2 & 3 & 4 & 2 & 1\end{array}\right)$ & $\mathrm{d}$ \\
$\left(\begin{array}{llllll}1 & 2 & 3 & 4 & 3 & 1\end{array}\right)$ & $\mathrm{e}$ \\
$\left(\begin{array}{llllll}1 & 2 & 3 & 4 & 3 & 2\end{array}\right)$ & $\mathrm{f}$ \\
\hline
\end{tabular}




\section{REFERENCES}

[1] B. Boe and D. H. Collingwood, A multiplicity one theorem for holomorphically induced representations, Math. Zeit., 192 (1986), 265-282.

[2] Intertwining operators between holomorphically induced modules, Pacific J. Math., 124 (1986), 73-84.

[3] B. Boe and T. J. Enright, Determination of the intertwining operators for holomorphically induced representations of SU( $p, q)$, Math. Ann., 275 (1986), 401-404.

[4] D. H. Collingwood, The n-homology of Harish-Chandra modules: generalizing a theorem of Kostant, Math. Ann., 272 (1985), 161-187.

[5] D. H. Collingwood, R. S. Irving and B. Shelton, Filtrations on holomorphically induced modules, to appear in J. Reine Angew. Math.

[6] T. J. Enright and B. Shelton, Decompositions in categories of highest weight modules, J. Algebra, 100 (1986), 380-402.

[7] _ Categories of highest weight modules: applications to classical Hermitian symmetric pairs, to appear in Mem. Amer. Math. Soc.

[8] R. Irving, Projective modules in the category $\mathcal{O}_{S}$ : Loewy series, Trans. Amer. Math. Soc., 291 (1985), 733-754.

[9] H. P. Jakobsen, Basic covariant differential operators on Hermitian symmetric spaces, Ann. Sci. École Norm. Sup., 18 (1985), 421-436.

[10] D. Vogan, A generalized $\tau$-invariant for the primitive spectrum of a semi-simple Lie algebra, Math. Ann., 242 (1979), 209-224.

Received August 22, 1986. The first author was supported in part by NSF grant \# DMS-8513467. The second author was supported in part by NSF grant \# DMS-8300793, and the third author was supported in part by NSF postdoctoral fellowship \#DMS8414100 .

University of California, Berkeley

BERKELEY, CA 94720

University of California, San Diego

LA Jolla, CA 92093

AND

UNIVERSITY OF OREGON

EUGENE, OR 97403 


\section{PACIFIC JOURNAL OF MATHEMATICS EDITORS}

\author{
V. S. VARADARAJAN \\ (Managing Editor) \\ University of California \\ Los Angeles, CA 90024 \\ Herbert Clemens \\ University of Utah \\ Salt Lake City, UT 84112 \\ R. FINN \\ Stanford University \\ Stanford, CA 94305
}

ROBION KIRBY

University of California

Berkeley, CA 94720

C. C. MOORE

University of California

Berkeley, CA 94720

HAROLD STARK

University of California, San Diego

La Jolla, CA 92093

\section{ASSOCIATE EDITORS}
R. ARENS
E. F. BECKENBACH
B. H. NEUMANN
F. WOLF
K. YOSHIDA (1906-1982)

\section{SUPPORTING INSTITUTIONS}
UNIVERSITY OF ARIZONA
UNIVERSITY OF OREGON
UNIVERSITY OF BRITISH COLUMBIA UNIVERSITY OF SOUTHERN CALIFORNIA
CALIFORNIA INSTITUTE OF TECHNOLOGY
UNIVERSITY OF CALIFORNIA
STANFORD UNIVERSITY
MONTANA STATE UNIVERSITY
UNIVERSITY OF HAWAII
UNIVERSITY OF NEVADA, RENO
UNIVERSITY OF TOKYO
NEW MEXICO STATE UNIVERSITY
UNIVERSITY OF UTAH
OREGON STATE UNIVERSITY
WASHINGTON STATE UNIVERSITY UNIVERSITY OF WASHINGTON 


\section{Pacific Journal of Mathematics}

\section{Vol. 131, No. $1 \quad$ November, 1988}

Tomek Bartoszynski, On covering of real line by null sets $\ldots \ldots \ldots \ldots \ldots 1$

Allen Davis Bell and Kenneth R. Goodearl, Uniform rank over differential operator rings and Poincaré-Birkhoff-Witt extensions $\ldots \ldots \ldots \ldots \ldots 13$

Brian Boe, Thomas Jones Enright and Brad Shelton, Determination of the intertwining operators for holomorphically induced representations of Hermitian symmetric pairs

Robert F. Brown, Topological identification of multiple solutions to

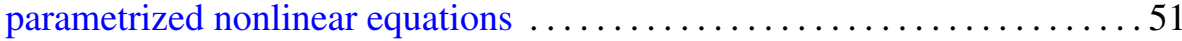

Marc R. M. Coppens, Weierstrass points with two prescribed nongaps ......71

Peter Larkin Duren and M. Schiffer, Grunsky inequalities for univalent functions with prescribed Hayman index .................... 105

Robert Greene and Hung-Hsi Wu, Lipschitz convergence of Riemannian manifolds

Kathryn E. Hare, Arithmetic properties of thin sets

Neal I. Koblitz, Primality of the number of points on an elliptic curve over a

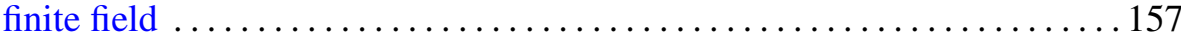

Isabel Dotti de Miatello and Roberto Jorge Miatello, Transitive isometry groups with noncompact isotropy

Raymond A Ryan, Weakly compact holomorphic mappings on Banach

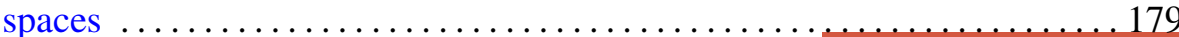

Tudor Zamfirescu, Curvature properties of typical convex surfaces 